\title{
Noncommutative geometry and the standard model
}

Encyclopedia of Mathematical Physics, J.-P. Franoise, G. Naber \& Tsou Sheung Tsun (eds.), Elsevier Science

\section{Introduction}

The aim of this contribution is to explain how Connes derives the standard model of electromagnetic, weak and strong forces from noncommutative geometry. The reader is supposed to be aware of two other derivations in fundamental physics: the derivation of the Balmer-Rydberg formula for the spectrum of the hydrogen atom from quantum mechanics and Einstein's derivation of gravity from Riemannian geometry.

At the end of the 19th century, new physics was discovered in atoms, their discrete spectra. Balmer and Rydberg succeeded to put order into the fast growing set of experimental numbers with the help of a phenomenological ansatz for the frequencies $\nu$ of the spectral rays of e.g. the hydrogen atom,

$$
\nu=g\left(n_{2}^{q}-n_{1}^{q}\right), \quad n_{j} \in \mathbb{N}, \quad q \in \mathbb{Z}, \quad g \in \mathbb{R} .
$$

The integer variables $n_{1}$ and $n_{2}$ reflect the discreteness of the spectrum. On the other hand the discrete parameter $q$ and the continuous parameter $g$ were fitted by experiment: $q=-2$ and $g=3.28910^{15} \mathrm{~Hz}$, the famous Rydberg constant. Later quantum mechanics was discovered and allowed to derive the Balmer-Rydberg ansatz and to constrain its parameters:

$$
q=2 \quad \text { and } \quad g=\frac{m_{e}}{4 \pi \hbar^{3}} \frac{e^{4}}{\left(4 \pi \epsilon_{0}\right)^{2}},
$$

in beautiful agreement with the anterior experimental fit.

\section{The standard model}

We propose to introduce the standard model in analogy with the Balmer-Rydberg formula, Tab. 1.

\subsection{The Yang-Mills-Higgs ansatz}

The variables of this Lagrangian ansatz are spin 1 particles $A$, spin $\frac{1}{2}$ particles decomposed into left- and right-handed components $\psi=\left(\psi_{L}, \psi_{R}\right)$ and spin 0 particles $\varphi$. There are four discrete parameters, a compact real Lie group $G$, and three unitary representations on complex Hilbert spaces $\mathcal{H}_{L}, \mathcal{H}_{R}$, and $\mathcal{H}_{S}$. The spin 1 particles come in a multiplet living in the complexified of the Lie algebra of $G, A \in \operatorname{Lie}(G)^{\mathbb{C}}$. The left-handed and right-handed spinors come in multiplets living in the Hilbert spaces, $\psi_{L} \in \mathcal{H}_{L}, \psi_{R} \in \mathcal{H}_{R}$. The (Higgs) scalar is another 
multiplet, $\varphi \in \mathcal{H}_{S}$. The Yang-Mills-Higgs Lagrangian together with its Feynman diagrams is spelled out in Tab. 2.

There are several continuous parameters: the gauge coupling $g \in \mathbb{R}_{+}$, the Higgs selfcouplings $\lambda, \mu \in \mathbb{R}_{+}$and a bunch of Yukawa couplings $g_{Y} \in \mathbb{C}$.

Let us choose $G=U(1) \ni e^{i \theta}$. Its irreducible unitary representations are all 1-dimensional, $\mathcal{H}=\mathbb{C} \ni \psi$ characterized by the charge $q \in \mathbb{Z}: \rho\left(e^{i \theta}\right) \psi=e^{i q \theta} \psi$. Then with $q_{L}=q_{R}$ and $\mathcal{H}_{S}=\{0\}$ we get Maxwell's theory with the photon (or gauge boson or 4-potential) $A$ coupled to the Dirac theory of a massless spinor of electric charge $q_{L}$ whose (relativistic) wave function is $\psi$. The gauge coupling is given by $g=e / \sqrt{\epsilon_{0}}$. Gauge invariance of the Yang-Mills-Higgs Lagrangian implies via Emmy Noether's theorem electric charge conservation in this case.

Yang-Mills models are therefore simply nonAbelian generalizations of electromagnetism where the Abelian gauge group $U(1)$ is replaced by any compact real Lie group. We insist on compact because all irreducible unitary representations of compact groups are finite dimensional. Finally the Higgs scalar is added to give masses to spinors and gauge bosons via spontaneous symmetry breaking.

We use compact groups and unitary representations as (discrete) parameters. One motivation is Noether's theorem and conserved quantities. The other comes from Wigner's theorem: The irreducible unitary representations of the Poincaré group are classified by mass and spin. Its orthonormal basis vectors are classified by energy-momentum and by the $z$-component of angular momentum. This theorem leads to the widely accepted definition of a particle as an orthonormal basis vector in a Hilbert space $\mathcal{H}$ carrying a unitary representation $\rho$ of a group G.

A precious property of the Yang-Mills-Higgs ansatz is its perturbative renormalizability necessary for fine structure calculations like the anomalous magnetic moment of the muon.

\subsection{The experimental fit}

Physicists have spent some thirty years and some $10^{9}$ Swiss Francs to distill the fit [1]:

$$
\begin{aligned}
G & =S U(2) \times U(1) \times S U(3) /\left(\mathbb{Z}_{2} \times \mathbb{Z}_{3}\right), \\
\mathcal{H}_{L} & =\bigoplus_{1}^{3}\left[\left(2, \frac{1}{6}, 3\right) \oplus\left(2,-\frac{1}{2}, 1\right)\right], \\
\mathcal{H}_{R} & =\bigoplus_{1}^{3}\left[\left(1, \frac{2}{3}, 3\right) \oplus\left(1,-\frac{1}{3}, 3\right) \oplus(1,-1,1)\right], \\
\mathcal{H}_{S} & =\left(2,-\frac{1}{2}, 1\right) .
\end{aligned}
$$


Here $\left(n_{2}, y, n_{3}\right)$ denotes the tensor product of an $n_{2}$ dimensional representation of $S U(2)$, '(weak) isospin', an $n_{3}$ dimensional representation of $S U(3)$, 'colour', and the one dimensional representation of $U(1)$ with 'hyper'charge $y$. For historical reasons the hypercharge is an integer multiple of $\frac{1}{6}$. This is irrelevant: in the Abelian case, only the product of the hypercharge with its gauge coupling is measurable and we do not need multi-valued representations, which are characterized by non-integer, rational hypercharges. In the direct sum, we recognize the three generations of fermions, the quarks, 'up, down, charm, strange, top, bottom', are $S U(3)$ triplets, the leptons, 'electron, $\mu, \tau$ ' and their neutrinos, are colour singlets. The basis of the fermion representation space is

$$
\begin{gathered}
\left(\begin{array}{l}
u \\
d
\end{array}\right)_{L},\left(\begin{array}{l}
c \\
s
\end{array}\right)_{L},\left(\begin{array}{l}
t \\
b
\end{array}\right)_{L},\left(\begin{array}{c}
\nu_{e} \\
e
\end{array}\right)_{L},\left(\begin{array}{c}
\nu_{\mu} \\
\mu
\end{array}\right)_{L},\left(\begin{array}{c}
\nu_{\tau} \\
\tau
\end{array}\right)_{L} \\
u_{R}, \quad c_{R}, \quad t_{R}, \quad e_{R}, \quad \mu_{R}, \quad \tau_{R} \\
d_{R}, \quad s_{R}, \quad b_{R},
\end{gathered}
$$

The parentheses indicate isospin doublets.

The eight gauge bosons associated to su(3) are called gluons. Attention, the $U(1)$ is not the one of electric charge, it is called hypercharge, the electric charge is a linear combination of hypercharge and weak isospin. This mixing is necessary to give electric charges to the $W$ bosons. The $W^{+}$and $W^{-}$are pure isospin states, while the $Z^{0}$ and the photon are (orthogonal) mixtures of the third isospin generator and hypercharge.

As the group $G$ contains three simple factors there are three gauge couplings,

$$
g_{2}=0.6518 \pm 0.0003, \quad g_{1}=0.3574 \pm 0.0001, \quad g_{3}=1.218 \pm 0.01
$$

The Higgs couplings are usually expressed in terms of of the $W$ - and Higgs-masses:

$$
\begin{aligned}
m_{W} & =\frac{1}{2} g_{2} v=80.419 \pm 0.056 \mathrm{GeV} \\
m_{\varphi} & =2 \sqrt{2} \sqrt{\lambda} v>98 \mathrm{GeV}
\end{aligned}
$$

with the vacuum expectation value $v:=\frac{1}{2} \mu / \sqrt{\lambda}$. Because of the high degree of reducibility of the spin $\frac{1}{2}$ representations there are 27 complex Yukawa couplings. They constitute the fermionic mass matrix which contains the fermion masses and mixings.

$$
\begin{array}{rrr}
m_{e}=0.510998902 \pm 0.000000021 \mathrm{MeV}, & m_{u}=3 \pm 2 \mathrm{MeV}, & m_{d}=6 \pm 3 \mathrm{MeV} \\
m_{\mu}=0.105658357 \pm 0.000000005 \mathrm{GeV}, & m_{c}=1.25 \pm 0.1 \mathrm{GeV}, & m_{s}=0.125 \pm 0.05 \mathrm{GeV}, \\
m_{\tau}=1.77703 \pm 0.00003 \mathrm{GeV}, & m_{t}=174.3 \pm 5.1 \mathrm{GeV}, & m_{b}=4.2 \pm 0.2 \mathrm{GeV}
\end{array}
$$


For simplicity, we have taken massless neutrinos. Then mixing only occurs for quarks and is given by a unitary matrix, the Cabibbo-Kobayashi-Maskawa matrix

$$
C_{K M}:=\left(\begin{array}{ccc}
V_{u d} & V_{u s} & V_{u b} \\
V_{c d} & V_{c s} & V_{c b} \\
V_{t d} & V_{t s} & V_{t b}
\end{array}\right)
$$

whose matrix elements in absolute value are:

$$
\left(\begin{array}{ccc}
0.9750 \pm 0.0008 & 0.223 \pm 0.004 & 0.004 \pm 0.002 \\
0.222 \pm 0.003 & 0.9742 \pm 0.0008 & 0.040 \pm 0.003 \\
0.009 \pm 0.005 & 0.039 \pm 0.004 & 0.9992 \pm 0.0003
\end{array}\right)
$$

The physical meaning of the quark mixings is the following: when a sufficiently energetic $W^{+}$

decays into a $u$ quark, this $u$ quark is produced together with a $\bar{d}$ quark with probability $\left|V_{u d}\right|^{2}$, together with a $\bar{s}$ quark with probability $\left|V_{u s}\right|^{2}$, together with a $\bar{b}$ quark with probability $\left|V_{u b}\right|^{2}$.

The phenomenological success of the standard model is phenomenal: with only a handful of parameters, it reproduces correctly some millions of experimental numbers: cross sections, life times, branching ratios.

\section{Noncommutative geometry}

Noncommutative geometry is an analytical geometry generalizing three other ones that also had important impact on our understanding of forces and time. Let us start by briefly recalling the three forerunners, Tab. 3. Euclidean geometry is underlying Newton's mechanics as space of positions. Forces are described by vectors living in the same space and the Euclidean scalar product is needed to define work and potential energy. Time is not part of geometry, it is absolute. This point of view is abandoned in special relativity unifying space and time into Minkowskian geometry. This new point of view allows to derive the magnetic field from the electric field as a pseudo force associated to a Lorentz boost. Although time has become relative, one can still imagine a grid of synchronized clocks, i.e. a universal time. The next generalization is Riemannian geometry = curved spacetime. Here gravity can be viewed as the pseudo force associated to a uniformly accelerated coordinate transformation. At the same time, universal time loses all meaning and we must content ourselves with proper time. With today's precision in time measurement, this complication of life becomes a bare necessity, e.g. the global positioning system (GPS).

Our last generalization is noncommutative geometry = curved space(time) with an uncertainty principle. As in quantum mechanics, this uncertainty principle is introduced via noncommutativity. 


\subsection{Quantum mechanics}

Consider the classical harmonic oscillator. Its phase space is $\mathbb{R}^{2}$ with points labelled by position $x$ and momentum $p$. A classical observable is a differentiable function on phase space such as the total energy $p^{2} /(2 m)+k x^{2}$. Observables can be added and multiplied, they form the algebra $\mathcal{C}^{\infty}\left(\mathbb{R}^{2}\right)$, which is associative and commutative. To pass to quantum mechanics, this algebra is rendered noncommutative by means of a noncommutation relation for the generators $x$ and $p:[x, p]=i \hbar 1$. Let us call $\mathcal{A}$ the resulting algebra 'of quantum observables'. It is still associative, has an involution .* (the adjoint or Hermitian conjugation) and a unit 1.

Of course, there is no space anymore of which $\mathcal{A}$ is the algebra of functions. Nevertheless, we talk about such a 'quantum phase space' as a space that has no points or a space with an uncertainty relation. Indeed, the noncommutation relation implies Heisenberg's uncertainty relation $\Delta x \Delta p \geq \hbar / 2$ and tells us that points in phase space lose all meaning, we can only resolve cells in phase space of volume $\hbar / 2$, see Fig. 1 . To define the uncertainty $\Delta a$ for an observable $a \in \mathcal{A}$, we need a faithful representation of the algebra on a Hilbert space, i.e. an injective homomorphism $\rho$ from $\mathcal{A}$ into the algebra of operators on $\mathcal{H}$. For the harmonic oscillator, this Hilbert space is $\mathcal{H}=\mathcal{L}^{2}(\mathbb{R})$. Its elements are the wave functions $\psi(x)$, square integrable functions on configuration space. Finally, the dynamics is defined by the Hamiltonian, a self adjoint observable $H=H^{*} \in \mathcal{A}$ via Schrödinger's equation $(i \hbar \partial / \partial t-\rho(H)) \psi(t, x)=0$. Here time is an external parameter, in particular, time is not an observable. This is different in the special relativistic setting where Schrödinger's equation is replaced by Dirac's equation $\not \partial \psi=0$. Now the wave function $\psi$ is the four-component spinor consisting of left- and righthanded, particle and antiparticle wave functions. The Dirac operator is not in $\mathcal{A}$ anymore, it is still an operator on $\mathcal{H}$. In Euclidean spacetime, the Dirac operator is also self adjoint, $\not^{*}=\not \partial$.

\section{$3.2 \quad$ Spectral triples}

Noncommutative geometry [2] does to a compact Riemannian spin manifold $M$ what quantum mechanics does to phase space. A noncommutative geometry is defined by the three purely algebraic items, $(\mathcal{A}, \mathcal{H}, \not \partial)$ called a spectral triple. $\mathcal{A}$ is a real, associative, possibly noncommutative involution algebra with unit, faithfully represented on a complex Hilbert space $\mathcal{H}$, and $\not \partial$ is a self adjoint operator on $\mathcal{H}$. As the spectral triple, also the axioms linking its three items are motivated by relativistic quantum mechanics. Connes reconstruction theorem (1996, 3]) states that there is a one-to-one correspondence between spectral triples with commutative algebra $\mathcal{A}$ and Riemannian manifolds $M$. As for classical phase space, the algebra consists of differentiable functions now on the Riemannian manifold, $\mathcal{A}=\mathcal{C}^{\infty}(M)$. The algebra is represented on spinors on which the Dirac operator acts. As for quantum phase space, Connes defines a non- 
commutative geometry by a spectral triple whose algebra is allowed to be noncommutative and he shows how important properties like dimensions, direct products, differentiation, integration and distances generalize to the noncommutative setting. As a bonus, the algebraic axioms of a spectral triple, commutative or not, include discrete i.e. 0-dimensional spaces that now are naturally equipped with a differential calculus. These spaces have finite dimensional algebras and Hilbert spaces meaning that their algebras are just matrix algebras.

\subsection{The spectral action}

In a next step Connes \& Chamseddine [3, 4] consider noncommutative spacetimes. They define the spectral action, a generalization of the Einstein-Hilbert action to noncommutative spacetimes, and compute it explicitly for almost commutative geometries.

An almost commutative geometry is defined as a direct product of a 4-dimensional commutative geometry, 'ordinary spacetime', by a 0-dimensional noncommutative geometry, the 'internal space'. If the latter is also commutative, e.g. the ordinary two point space, then the direct product describes a two-sheeted universe or a Kaluza-Klein space whose fifth dimension is discrete, [5]. In general, the axioms of spectral triples imply that the 'Dirac' operator of the internal space is precisely the fermionic mass matrix.

On almost commutative geometries, the spectral action is equal to the Einstein-Hilbert action plus the Yang-Mills-Higgs ansatz, Fig. 2. In other words, noncommutative geometry explains the forces mediated by gauge bosons and Higgs scalars as pseudo-forces accompanying the gravitational force in the same way that Minkowskian geometry (i.e. special relativity) explains the magnetic force as a pseudo-force accompanying the electric force.

There are constraints on the discrete and continuous parameters in the Yang-Mills-Higgs ansatz deriving from the spectral action Fig. 3 .

In particular if we consider only irreducible spectral triples and among them only those which produce non-degenerate fermion masses compatible with renormalization then we only get the standard model with one generation of quarks and leptons, with a massless neutrino and with an arbitrary number of colours, and a few submodels thereof. More than one generation and neutrino masses are possible but imply reducible triples. However in at least one generation, the neutrino must remain purely left and massless.

For the standard model with $N$ generations and $N_{c}$ colours, we have the constraints $g_{N_{c}}^{2}=$ $g_{2}^{2}=(9 / N) \lambda$ on the continuous parameters. If we put $N=N_{c}=3$ and if we believe in the popular 'big desert' then these constraints yield a 'unification scale' $\Lambda=10^{17} \mathrm{GeV}$ at which the uncertainty relation in spacetime should become manifest, $\Delta \tau=\hbar / \Lambda$, and a Higgs-mass of $m_{\varphi}=171.6 \pm 5 \mathrm{GeV}$ for $m_{t}=174.3 \pm 5.1 \mathrm{GeV}$, see Fig. 4 
It is clear that almost commutative geometries only scratch the surface of a gold mine. May we hope that a genuinely noncommutative geometry will solve our present problems with quantum field theory and quantum gravity??

\section{References}

[1] The Particle Data Group, Particle Physics Booklet and http://pdg.lbl.gov

[2] A. Connes, Noncommutative Geometry, Academic Press (1994)

A. Connes, Noncommutative geometry and reality, J. Math. Phys. 36 (1995) 6194

[3] A. Connes, Gravity coupled with matter and the foundation of noncommutative geometry, hep-th/9603053, Comm. Math. Phys. 155 (1996) 109

[4] A. Chamseddine \& A. Connes, The spectral action principle, hep-th/9606001, Comm. Math. Phys.186 (1997) 731

[5] J. Madore, An Introduction to Noncommutative Differential Geometry and Its Physical Applications, Cambridge University Press (1995)

[6] Further reading:

L. O'Raifeartaigh, Group Structure of Gauge Theories, Cambridge University Press (1986)

C. P. Martín, J. M. Gracia-Bondía \& J. C. Várilly, The standard model as a noncommutative geometry: the low mass regime, hep-th/9605001, Phys. Rep. 294 (1998) 363

G. Landi, An Introduction to Noncommutative Spaces and Their Geometry, hep-th/9701078, Springer (1997)

J. M. Gracia-Bondía, J. C. Várilly \& H. Figueroa, Elements of Noncommutative Geometry, Birkhäuser (2000)

D. Kastler, Noncommutative geometry and fundamental physical interactions: the Lagrangian level, J. Math. Phys. 41 (2000) 3867

F. Scheck, W. Werner \& H. Upmeier (eds.), Noncommutative Geometry and the Standard Model of Elementary Particle Physics, Lecture notes in physics 596, Springer (2002)

T. Schücker, Forces from Connes' geometry, in 'Topology and Geometry in Physics', eds.: E. Bick \& F. Steffen, hep-th/0111236, Lecture notes in physics, Springer, to appear 
Thomas Schücker

Centre de Physique Théorique

case 907, F-13288 Marseille, cedex 9

also at Université de Provence

schucker@cpt.univ-mrs.fr

\begin{tabular}{l|ll} 
& atomic physics & particle physics \\
\hline \multirow{2}{*}{$\begin{array}{l}\text { new physics } \\
\text { ansatz }\end{array}$} & $\begin{array}{ll}\text { discrete spectra } \\
\nu=g\left(n_{2}^{q}-n_{1}^{q}\right)\end{array}$ & forces mediated by gauge bosons \\
experimental fit & $q=-2, g=3.28910^{15} \mathrm{~Hz}$ & Yang-Mills-Higgs models \\
underlying theory & quantum mechanics & noncommutative geometry \\
& \multicolumn{2}{c}{ Table 1: An analogy }
\end{tabular}

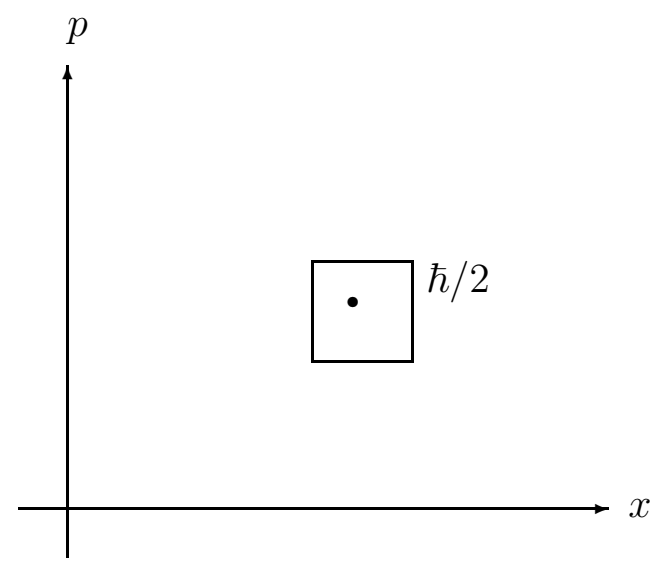

Figure 1: The first example of noncommutative geometry 


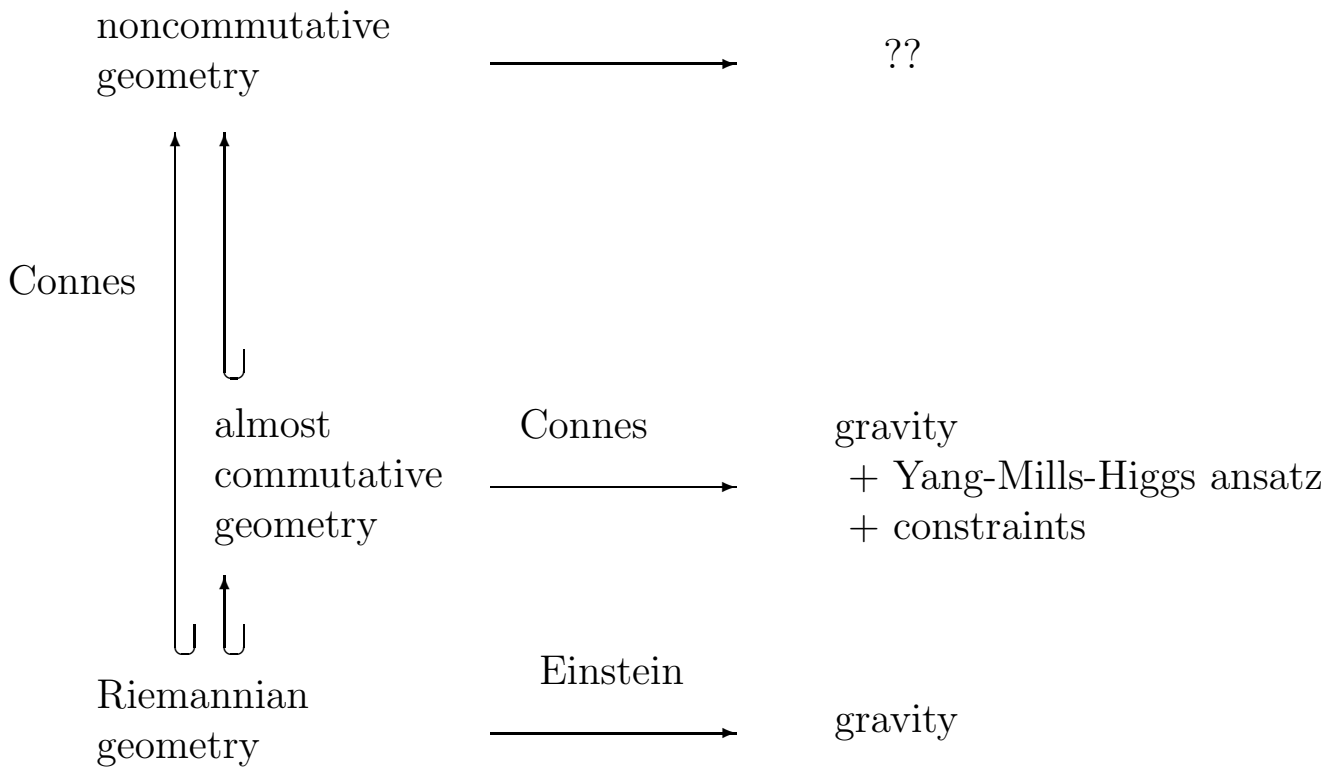

Figure 2: Deriving the Yang-Mills-Higgs ansatz from gravity

Yang-Mills-Higgs

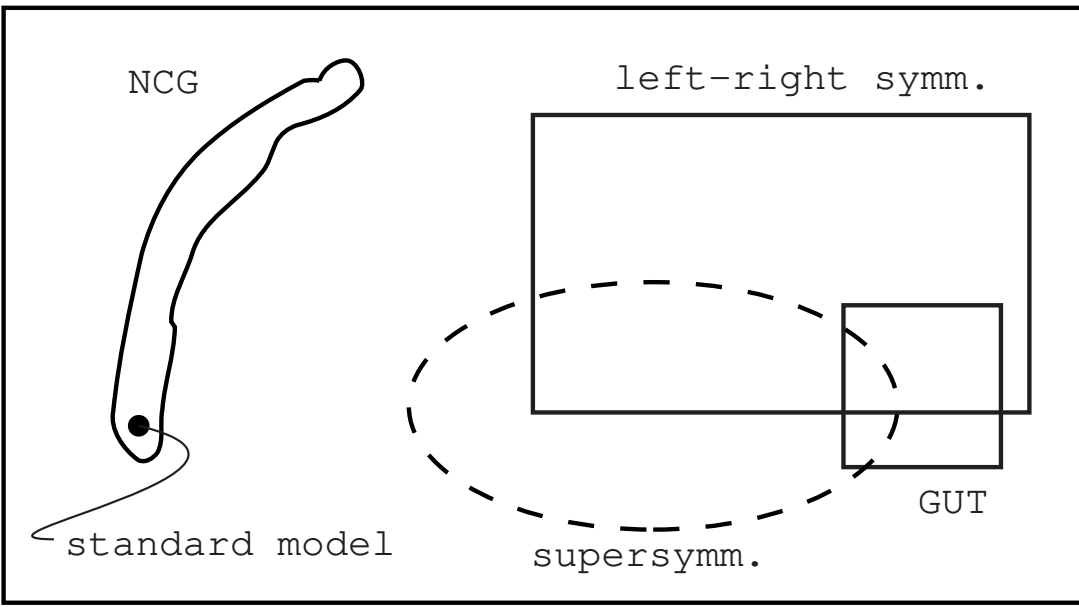

Figure 3: Constraints inside the ansatz 


$$
\begin{aligned}
\mathcal{L}[A, \psi, \varphi]= & \frac{1}{2} \operatorname{tr}\left(\partial_{\mu} A_{\nu} \partial^{\mu} A^{\nu}-\partial_{\mu} A_{\nu} \partial^{\nu} A^{\mu}\right) \\
& +g \operatorname{tr}\left(\partial_{\mu} A_{\nu}\left[A^{\mu}, A^{\nu}\right]\right) \\
& +g^{2} \operatorname{tr}\left(\left[A_{\mu}, A_{\nu}\right]\left[A^{\mu}, A^{\nu}\right]\right) \\
& +\bar{\psi} \not \partial \psi \\
& +i g \bar{\psi}\left(\tilde{\rho}_{L} \oplus \tilde{\rho}_{R}\right)\left(A_{\mu}\right) \gamma^{\mu} \psi \\
& +\frac{1}{2} \partial_{\mu} \varphi^{*} \partial^{\mu} \varphi \\
& +\frac{1}{2} g\left\{\left(\tilde{\rho}_{S}\left(A_{\mu}\right) \varphi\right)^{*} \partial^{\mu} \varphi+\partial_{\mu} \varphi^{*} \tilde{\rho}_{S}\left(A_{\mu}\right) \varphi\right\} \\
& +\frac{1}{2} g^{2}\left(\tilde{\rho}_{S}\left(A_{\mu}\right) \varphi\right)^{*} \tilde{\rho}_{S}\left(A^{\mu}\right) \varphi \\
& +\lambda \varphi^{*} \varphi \varphi^{*} \varphi
\end{aligned}
$$

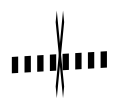

Table 2: The Yang-Mills-Higgs Lagrangian and its Feynman diagrams 


\begin{tabular}{l|ll} 
geometry & force & time \\
\hline \multirow{2}{*}{ Euclidean } & $E=\int \vec{F} \cdot \mathrm{d} \vec{x}$ & absolute \\
Minkowskian & $\vec{E}, \epsilon_{0} \Rightarrow \vec{B}, \mu_{0}=\frac{1}{\epsilon_{0} c^{2}}$ & universal \\
Riemannian & Coriolis $\leftrightarrow$ gravity & proper, $\tau$ \\
noncommutative & gravity $\Rightarrow \mathrm{YMH}, \lambda=\frac{1}{3} g_{2}^{2}$ & $\Delta \tau \sim 10^{-40} \mathrm{~s}$
\end{tabular}

Table 3: Four nested analytic geometries

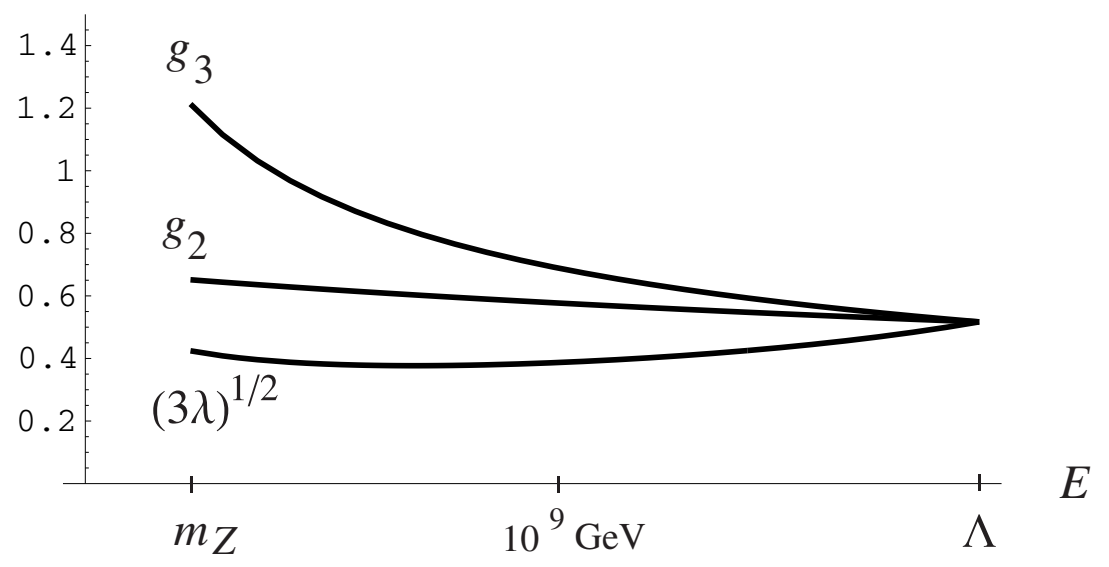

Figure 4: Running coupling constants 